\title{
High Pressure Raman Spectroscopy in Carbon Nanotubes
}

\author{
G.A. Kourouklis ${ }^{a}$, J. Arvanitidis ${ }^{a, b}$, D. Christofilos ${ }^{a}$ And S. Ves ${ }^{c}$ \\ ${ }^{a}$ Physics Division, School of Technology, Aristotle University of Thessaloniki \\ 54124 Thessaloniki, Greece \\ ${ }^{b}$ Department of Applied Sciences, Technological Educational Institute of Thessaloniki \\ 57400 Sindos, Greece \\ ${ }^{c}$ Physics Department, Aristotle University of Thessaloniki, 54124 Thessaloniki, Greece
}

\begin{abstract}
This work focuses on the high pressure Raman study of carbon nanostuctures comprising of single- or double-wall carbon nanotubes. The detailed examination of the Raman peaks, especially those attributed to the radial breathing modes of the carbon nanotubes, as a function of pressure provides a wealth of information concerning the pressure response of individual nanotubes as well as the internal-external tube (intratube) interactions. The radial breathing modes of both the internal and the external tubes in double-wall carbon nanotubes show reduced pressure slope values as compared to the corresponding in single-wall carbon nanotubes. The reduced slopes for the internal tubes reflect the pressure screening effect inside the external tubes, while those for the external tubes their structural reinforcement due to the encapsulation of smaller diameter tubes in their interior. Moreover, the magnitude of the pressure screening effect depends strongly on the intratube spacing and thus on the intratube interaction. All the experimental observations are well reproduced qualitatively by means of theoretical calculations based on a simple phenomenological model.
\end{abstract}

PACS numbers: 78.67.Ch, 78.30.Na, 63.22.-m, 62.50.-p

\section{Introduction}

In the last two decades, carbon nanotube materials consisting of one (single-wall carbon nanotubes, SWCNTs) or more coaxial rolled graphene layers (multi-wall carbon nanotubes, MWCNTs) have attracted the intense scientific interest worldwide thanks to their unique physical properties that provide a broad field for basic research and nanotechnology applications. Double-wall carbon nanotubes (DWCNTs) constitute a model system for studying encapsulation effects in carbon nanotube systems, while their mechanical and transport properties are determined by the structural characteristics of the constituent internal and external tubes and their mutual (intratube) interaction. High pressure application on carbon nanotube materials is a valuable tool for the investigation of their mechanical and structural stability and resonance Raman spectroscopy allows the selective probing of different nanotubes $[1,2]$. This is possible due to the size-dependent peculiar electronic structure of carbon nanotubes, originating from the quasi one-dimensional electron confinement $[3,4]$, in combination with the inversely proportional relation between the radial breathing mode ( $\mathrm{RBM})$ frequency and the tube diameter [5].

Theoretical calculations on bundled or individual SWCNTs suggest that the nanotubes suffer significant diameter dependent pressure induced cross-section deformations from their circular to oval or polygonized and eventually to flattened shapes, with nanotubes of smaller diameter exhibiting higher structural stability [6-8]. High pressure Raman studies reveal that these tube cross-section deformations cause the strong intensity attenuation and the disappearance of the RBM band at elevated pressures $[1,9,10]$. Moreover, the theoretically predicted diameter dependence of the nanotube cross-section deformation is also verified experimentally for both bundled and individual SWCNTs [11, 12]. On the other hand, theoretical calculations predict an enhancement of the structural rigidity of the nanotubes after the encapsulation of smaller diameter tubes in their interior and the formation of DWCNTs [13]. In line with this prediction, high pressure Raman studies on DWCNTs show that the presence of the internal tubes provide structural support to the externals against pressure application [14]. Moreover, the external tubes in DWCNTs act as a protection shield for the internals, resulting in the reduction of the effective pressure exerted on the latter $[14,15]$. The present work reviews our recent high pressure Raman studies on carbon nanotube materials, aiming to the clarification of the pressure response of the internal and the external tubes in DWCNTs as compared to that of the corresponding SWCNTs and the role of the intratube interaction in the stability of DWCNTs. The experimental findings are also combined with theoretical calculations based on a simple phenomenological model of two anharmonically coupled anharmonic oscillators. 


\section{Experimental details}

The starting bundled SWCNT material (tube diameters: $1.25-1.6 \mathrm{~nm}[16]$ ) was generated by laser vaporization of $\mathrm{Co}$ and $\mathrm{Ni}$ doped carbon rods in $\mathrm{Ar}$ atmosphere [17]. The intermediate step $\mathrm{C}_{60}$-peapods were prepared by the reaction of purified uncapped SWCNTs with $\mathrm{C}_{60}$ vapor, while the final DWCNT material has been synthesized by means of the peapod conversion process, following Bandow's procedure [18]. The Raman spectra of the studied carbon nanotube materials were recorded in the backscattering geometry using a micro-Raman triple grating system (DILOR XY), equipped with a cryogenic CCD detector. High pressure Raman measurements were carried out using a Mao-Bell type diamond anvil cell with the 4:1 methanol-ethanol mixture as the pressure transmitting medium (ensuring good hydrostatic conditions at least up to $10 \mathrm{GPa}$ ) and the ruby fluorescence technique for pressure calibration. For excitation, the $514.5 \mathrm{~nm}$ line of an $\mathrm{Ar}^{+}$laser, the $632.8 \mathrm{~nm}$ line of a He-Ne laser and the 647.1 and $676.4 \mathrm{~nm}$ lines of a $\mathrm{Kr}^{+}$laser have been alternatively used. The excitation line was focused on the sample by means of a $20 \times(100 \times$ at ambient pressure $)$ objective, while the laser power was kept below $2.5 \mathrm{~mW}$, measured before the cell (below $0.1 \mathrm{~mW}$ with the $100 \times$ objective), in order to eliminate laser-heating effects.

\section{Phenomenological model}

According to the phenomenological model employed in our study [19], the elastic properties of a carbon nanotube are approximated by those of its cross-section, modeled as a ring of carbon atoms in a regular polygonal arrangement interacting with each other through a Lennard-Jones (LJ) potential (equilibrium $\mathrm{C}-\mathrm{C}$ bond length: $0.142 \mathrm{~nm}$ ). The fully symmetric radial breathing-like mode (RBLM) and the in-plane tangential $\mathrm{G}^{-}$-like mode (GLM) vibrations of the atoms in the ring correspond to the RBM and the $\mathrm{G}^{-}$mode of a carbon nanotube, respectively. For infinitely large ring diameter the RBLM frequency tends to zero, while we require that the GLM frequency attains the value of the tangential mode of graphite $\left(1580 \mathrm{~cm}^{-1}\right)$, allowing thus the determination of the energy LJ parameter in the single-ring model. The pressure application in our model is approximated by radial stress.

The radial symmetry of the system allows the description of the ring by an equivalent anharmonic oscillator of LJ type with the same parameters to those determined for the ring, simplifying considerably the calculation of the RBLM frequency and its pressure response. A combination of two such anharmonic oscillators, interacting through another LJ potential with an equilibrium distance of $0.344 \mathrm{~nm}$ (the turbostratic constraint of graphite at ambient conditions), has been used to calculate the pressure dependence of the RBLM frequencies of the internal and the external rings approximating a DWCNT. The energy parameter of the LJ potential describing the internal-external ring (intraring) coupling is determined by assuming that for a large number of concentric rings with infinitely large length, the highest frequency RBLM mode should attain the frequency of the $B_{2 \mathrm{~g}}$ mode of graphite $\left(127 \mathrm{~cm}^{-1}\right)$.

\section{Results and discussion}

The Raman spectrum of the starting SWCNTs, the intermediate step $\mathrm{C}_{60}$-peapods and the resulting DWCNTs are illustrated in Fig. 1a. Apart from the low frequency RBMs, the Raman spectrum of the SWCNTs contains two more main bands in the depicted frequency region. The intermediate frequency band marked by " $D$ " is attributed to a disorder-induced mode, which is strongly diameter and excitation energy dependent [20]. The high frequency, particularly strong band marked by "G" corresponds to the in-plane carbon stretching vibrations perpendicular (lower frequency $\mathrm{G}^{-}$component) and parallel (higher frequency $\mathrm{G}^{+}$component) to the nanotube axis [21]. As can be seen from Fig. 1a, the encapsulation of the $\mathrm{C}_{60}$ molecules in the SWCNTs and their mutual interaction results to small frequency shifts of the Raman peaks and the split of the RBMs. Moreover, a new peak appears at $\approx 1465 \mathrm{~cm}^{-1}$ that is assigned to the $A_{\mathrm{g}}(2)$ intramolecular mode of $\mathrm{C}_{60}[22]$. In the final stage of the preparation process, the transformation from the $\mathrm{C}_{60}$-peapods to DWCNTs causes the appearance of new nanotube peaks and the disappearance of the $\operatorname{Ag}(2) \mathrm{C}_{60}$ mode, associated with the formation of the internal tubes from the encapsulated fullerene molecules. More specifically, the new Raman peaks emerging in the frequency region $300-400 \mathrm{~cm}^{-1}$ are attributed to the radial breathing modes of the internal tubes. In addition, a shoulder appears in the low frequency side of the D band and a relatively strong peak between the $\mathrm{G}^{-}$and the $\mathrm{G}^{+}$bands of the pristine SWCNTs, corresponding spectrum to the $\mathrm{D}\left(\mathrm{D}_{\mathrm{int}}\right)$ and the $\mathrm{G}$ band $\left(\mathrm{G}_{\mathrm{int}}^{+}\right)$of the smaller diameter internal tubes, respectively.

The ambient pressure Raman spectra in the RBM frequency region of the studied DWCNTs, excited by the laser wavelengths given in the experimental section, are illustrated in Fig. 1b. These Raman spectra differ significantly from each other regarding the number and the frequency of the various components as well as their relative intensity. This is explained by the fact that Raman spectroscopy in carbon nanotubes - as already mentioned above - allows the selective probing of specific tubes being in resonance with the excitation wavelength. According to the Kataura et al. plot [3], in which the energy separations of the Van Hove singularities in the 1D electron density of states (e-DOS) of the nanotubes are plotted as a function of their diameter $d$, the excitation of the studied DWCNTs with $514.5 \mathrm{~nm}$ probes mainly semiconducting internal and external tubes while the excitation with $632.8,647.1$ and $676.4 \mathrm{~nm}$ probes semiconducting internal and mainly metallic external tubes.

The Raman spectra in the RBM frequency region of the studied DWCNTs excited by the $514.5 \mathrm{~nm}$ wave- 

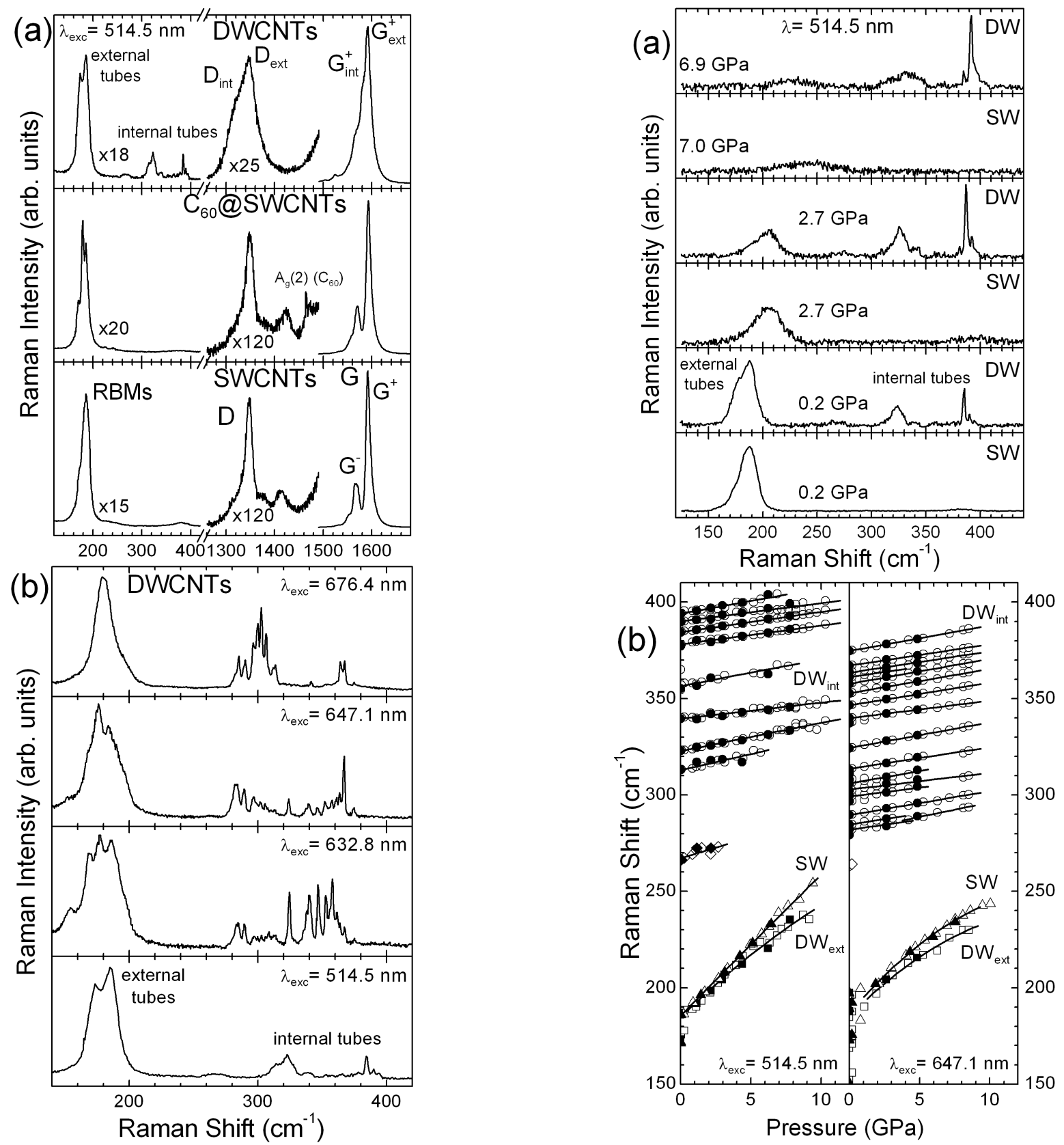

Fig. 1. (a) Raman spectra of SWCNTs, $\mathrm{C}_{60}$ at SWCNTs peapods and DWCNTs recorded at ambient conditions and excited with $514.5 \mathrm{~nm}$. The RBM and the D-band regions in all spectra have been suitably enhanced to improve visibility. (b) Raman spectra of DWCNTs in the RBM frequency region recorded at ambient conditions and excited with four different laser lines.

length and recorded at various pressures are illustrated in Fig. 2 along with the corresponding of the pristine SWCNTs. With increasing pressure, all the Raman peaks shift towards higher energies, while at the same time significant relative intensity changes take place. Namely, the RBM bands of the SWCNTs and the ex-

Fig. 2. (a) Raman spectra of SWCNTs (SW) and DWCNTs (DW) in the RBM frequency region excited by the $514.5 \mathrm{~nm}$ laser line and recorded at various pressures. (b) Pressure dependence of the RBM frequencies in SWCNTs (SW, triangles) and DWCNTs (DW ext for the external tubes, squares and $\mathrm{DW}_{\text {int }}$ for the internal tubes, circles) excited by the 514.5 and the $647.1 \mathrm{~nm}$ laser lines. Diamonds correspond to an RBM peak of DWCNTs for which an unambiguous assignment to internal or external tubes cannot be made [14]. Open (solid) symbols denote data taken for increasing (decreasing) pressure while solid lines are least squares fittings. 
ternal tubes in DWCNTs display strong intensity attenuation and broadening, reflecting the pressure-induced distortion of their cross-section that eliminates the radial band. On the other hand, the RBM band of the internal tubes in DWCNTs is hardly affected by the pressure and the constituent peaks remain intense and narrow up to the highest pressure reached in our experiments $(10.3 \mathrm{GPa})$. This experimental observation is a strong indication of the pressure screening effect of the internal tubes in DWCNTs due to their encapsulation in the interior of the externals. The pressure screening effect is also reflected in the pressure evolution of the internal tubes RBM frequencies as compared to that of the external tubes (Fig. 2b), where the pressure induced shift for the former is much smaller than that for the latter.

In Fig. $2 \mathrm{~b}$ the pressure dependence of the RBM frequencies in SWCNTs is also included for comparison. As can be seen from this figure, the pressure dependence for both the semiconducting (excitation with the $514.5 \mathrm{~nm}$ wavelength) and the metallic (excitation with the $647.1 \mathrm{~nm}$ wavelength) external tube RBMs is slightly less pronounced than that for the SWCNTs. This difference indicates the supporting effect in the case of the external tubes in DWCNTs due to the encapsulation of the smaller diameter internal tubes in their interior. As the intratube interaction in DWCNTs increases with pressure, the observed structural support effect becomes progressively stronger, resulting to a more pronounced sublinear pressure response of external tube RBMs than that of the SWCNTs.

In Fig. 3 we compile and compare the experimentally obtained normalized pressure slopes, $\Gamma_{i}=$ $\left(1 / \omega_{i}\right)\left(\partial \omega_{i} / \partial P\right)$, of the SWCNT and the DWCNT RBMs as a function of their frequency (inversely proportional to the tube diameter in SWCNT [5]). We include also data taken from the literature for different than those studied here bundled and individual SWCNTs [11, 12] and bundled DWCNTs $[23,24]$. The $\Gamma$ parameter values of the external tubes in DWCNT exhibit strong and similar frequency (and thus diameter) dependence on that of SWCNTs. Namely, they decrease quasi-quadratically with decreasing tube diameter, which is rationalized in terms of the enhanced cross-section fragility of the larger nanotubes upon pressure [11]. Moreover, in agreement with the discussion made above, the $\Gamma$ parameters for the SWCNTs are slightly larger than those of the same tubes used as externals in DWCNTs, reflecting the structural support of the latter provided by the internal tubes.

The $\Gamma$ parameter values obtained for the internal tubes in DWCNTs are much smaller than those for the similar diameter SWCNTs, in agreement with the pressure screening effect due to their encapsulation in the externals. They also exhibit an overall decrease with increasing $\omega$, which again is attributed to the higher stiffness of the smaller diameter tubes. Nevertheless, the data in this case are distributed in five groups, in which $\Gamma_{i}$ increases with $\omega_{i}$. Let us note that the data taken from the literature lie, within their experimental uncertainty, on the
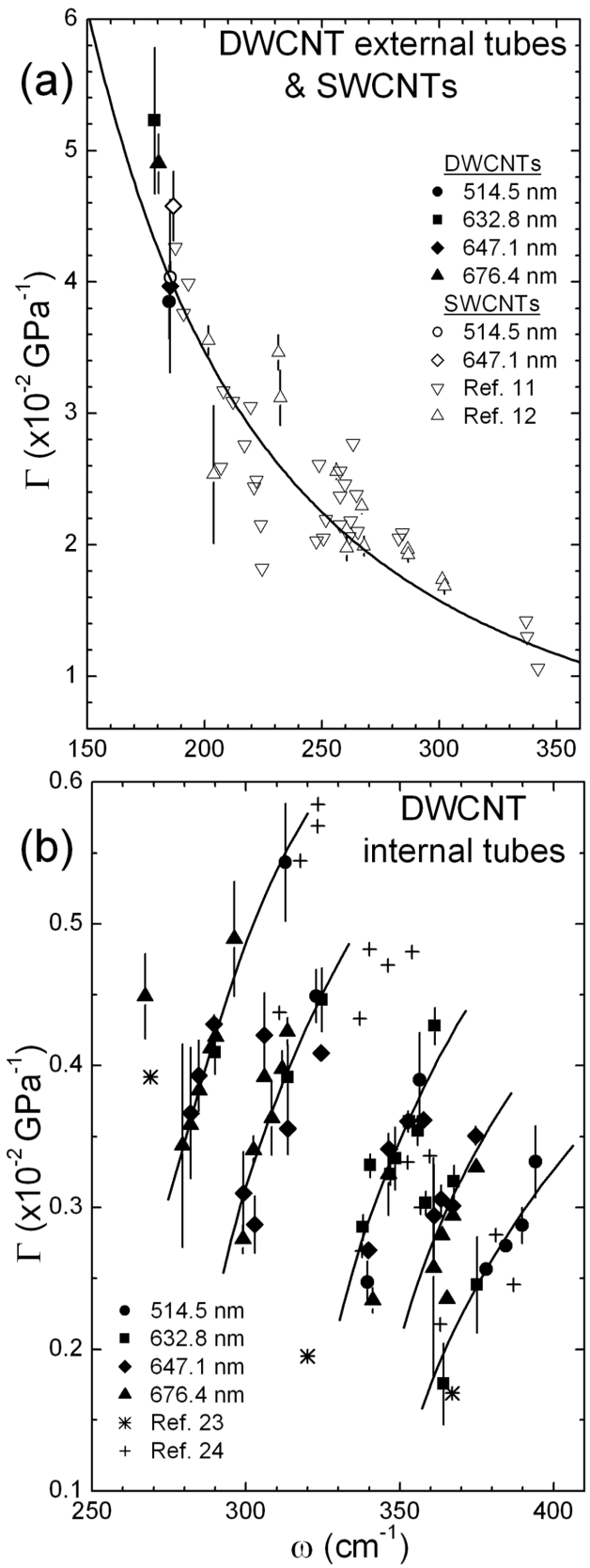

Fig. 3. Experimental normalized pressure slopes, $\Gamma_{i}=$ $\left(1 / \omega_{i}\right)\left(\partial \omega_{i} / \partial P\right)$ of the RBMs in (a) SWCNTs (open symbols), DWCNT external tubes (solid symbols) and (b) DWCNT internal tubes as a function of their frequency. Triangles in the top part (corresponding to two different SWCNT samples produced by the HiPCO process), as well as crosses and asterisks in the bottom part (corresponding to two different DWCNT samples produced through the $\mathrm{C}_{60}$ peapod conversion route) are data taken from the literature $[11,12,23,24]$. The solid lines through the data are guides to the eye. 

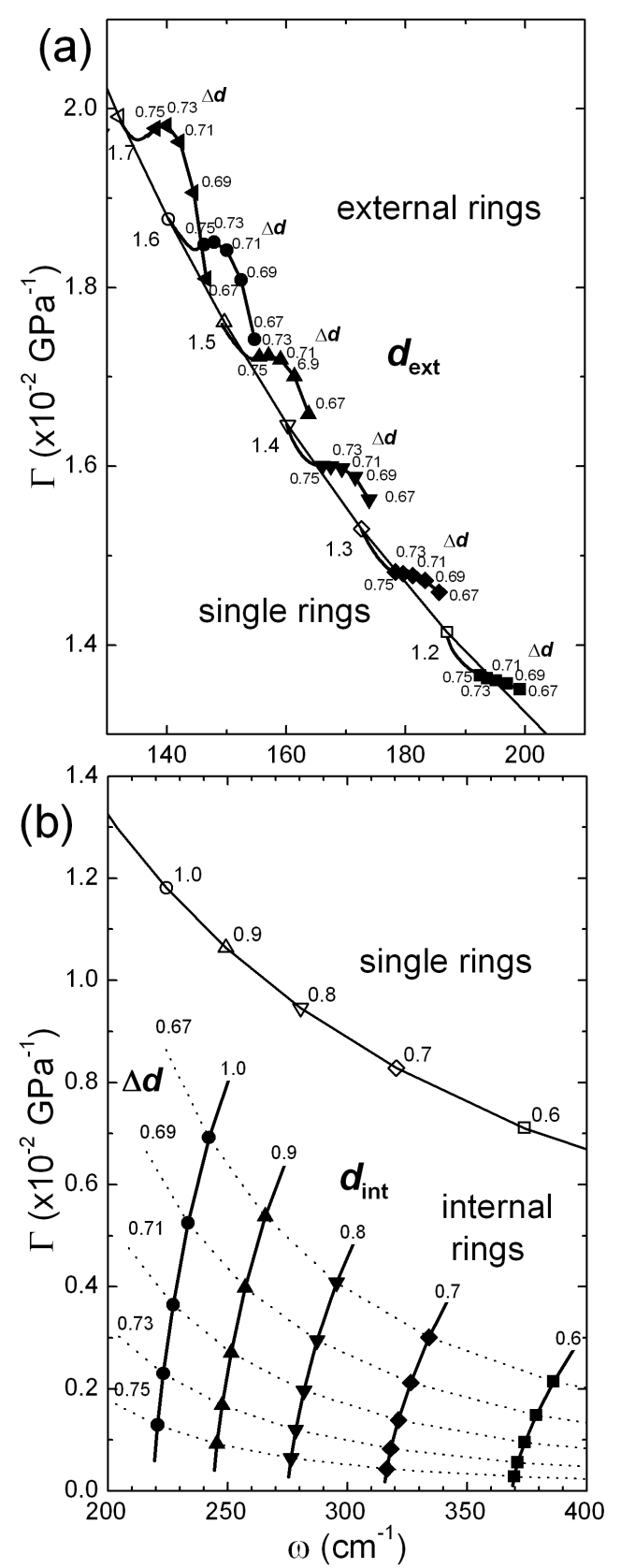

Fig. 4. Calculated normalized pressure slopes, $\Gamma_{i}=$ $\left(1 / \omega_{i}\right)\left(\partial \omega_{i} / \partial P\right)$, of the RBLMs of the single rings (open symbols) and (a) the external or (b) the internal rings comprising the double ring model system (solid symbols). Thin solid lines connect data corresponding to the single rings, thick solid lines group data originating from (a) the same external ring of diameter $d_{\text {out }}$ combined with internal rings of different diameters $d_{\text {out }}$ or (b) the same internal ring combined with different diameter externals, while the dotted lines in the right panel delineate $\Gamma_{i}$ values for internal-external pairs of constant $\Delta d=d_{\text {out }}-d_{\text {in }}$. All lengths in the figures are expressed in $\mathrm{nm}$. groups constructed from the $\Gamma$ parameter values of the studied DWCNT material. The observed behavior could be understood taking into account that for a specific internal tube, several compatible externals may exist, leading to very close intratube spacings, but enough to cause an observable split of the RBMs in the Raman spectrum [25]. In the split components, the better the tube matching, the higher the frequency and the higher the corresponding $\Gamma$ parameter value. Therefore, each of the five groups illustrated in Fig. 3b could be ascribed to the same (or different with similar diameters) internal tube encapsulated in external tubes of different diameters.

In the framework of the phenomenological model presented in the previous section, it is feasible to construct also the corresponding $\Gamma_{i}-\omega_{i}$ plot for external and internal ring combinations of different diameters $d_{\text {ext }}$ and $d_{\text {int }}$, respectively. In Fig. 4 the calculated values are plotted for the external (internal) rings with $d_{\text {ext }}=1.2-1.7 \mathrm{~nm}$ $\left(d_{\text {int }}=0.6-1.0 \mathrm{~nm}\right)$ and for various inner-outer ring diameter differences $(\Delta d)$ in the range $0.67-0.75 \mathrm{~nm}$, compatible with the intratube spacing $0.34-0.38 \mathrm{~nm}$ of the studied DWCNT sample [26]. Data calculated for single rings with the same diameters to the external and the internal rings are also included for comparison. Despite the apparent simplicity of the model employed, leading to deviation between the calculated and experimental $\Gamma$ values, their order of magnitude and their overall trend are the same. More specifically, as can be inferred from Fig. 4a, the calculated $\Gamma$ parameter values for the external rings lie closely to the corresponding values for single rings, which decrease with increasing $\omega$ (decreasing tube diameter), reflecting the larger stiffness of the smaller tubes. However, the $\Gamma$ parameter values for the external rings are systematically smaller than those of the single rings and decrease monotonically with decreasing $\Delta d$ (increasing intraring interaction). This behavior indicates the structural support of the external ring against pressure provided by the presence of the internal and underlines the significant role played by the intratube interaction in the structural stability of the DWCNTs.

The $\Gamma$ parameters values for the internal rings are also smaller than those for the corresponding single rings, indicating in this case the pressure screening in the interior of the external tubes in DWCNTs. More importantly, our calculations reproduce qualitatively the grouping behavior of the experimental $\Gamma_{i}-\omega_{i}$ data for the internal tubes of DWCNTs. Namely, for a specific internal ring, the decrease of $\Delta d$ causes the increase of the intraring interaction, resulting in the upshift of the RBLM frequency and the more efficient pressure transmission to the inner ring (larger $\Gamma$ parameter value). It is then evident that the intratube interaction determines the effective pressure applied to the internal tubes in DWCNTs and verifies the assumption that the grouping of the experimental $\Gamma_{i}-\omega_{i}$ data can be attributed to the same internal tube (or different with similar diameters) encapsulated into external tubes of different diameter. 


\section{Conclusions}

Summarizing, our high pressure Raman studies of DWCNTs and their parent SWCNTs combined with model calculations reveal the structural support of the external tubes provided by the presence of their internals through the intratube interaction and the pressure screening effect of the latter due to their encapsulation inside the former. The intratube interaction determines also the magnitude of the pressure screening effect in the interior of the external tubes in DWCNTs.

\section{References}

[1] I. Loa, J. Raman Spectrosc. 34, 61 (2003).

[2] M.S. Dresselhaus, P.C. Eklund, Adv. Phys. 49, 705 (2000).

[3] H. Kataura, Y. Kumazawa, Y. Maniwa, I. Umezu, S. Suzuki, Y. Ohtsuka, Y. Achiba, Synth. Metals 103, 2555 (1999).

[4] F. Wang, G. Dukovic, L.E. Brus, T.F. Heinz, Science 308, 838 (2005).

[5] A.M. Rao, E. Richter, S. Bandow, B. Chase, P.C. Eklund, K.A. Williams, S. Fang, K.R. Subbaswamy, M. Menon, A. Thess, R.E. Smalley, G. Dresselhaus, M.S. Dresselhaus, Science 275, 187 (1997).

[6] S.-P. Chan, W.-L. Yim, X.G. Gong, Z.-F. Liu, Phys. Rev. B 68, 075404 (2003).

[7] J.A. Elliott, J.K.W. Sandler, A.H. Windle, R.J. Young, M.S.P. Shaffer, Phys. Rev. Lett. 92, 095501 (2004).

[8] P. Tangney, R.B. Capaz, C.D. Spataru, M.L. Cohen, S.G. Louie, Nano Lett. 5, 2268 (2005).

[9] U.D. Venkateswaran, A.M. Rao, E. Richter, M. Menon, A. Rinzler, R.E. Smalley, P.C. Eklund, Phys. Rev. B 59, 10928 (1999).

[10] A. Merlen, N. Bendiab, P. Toulemonde, A. Aouizerat, A. San Miguel, J.-L. Sauvajol, G. Montagnac, H. Cardon, P. Petit, Phys. Rev. B 72, 035409 (2005).

[11] U.D. Venkateswaran, D.L. Masica, G.U. Sumanasekara, C.A. Furtado, U.J. Kim, P.C. Eklund, Phys. Rev. B 68, 241406 (2003).
[12] D. Christofilos, J. Arvanitidis, K.S. Andrikopoulos, G.A. Kourouklis, S. Ves, T. Takenobu, Y. Iwasa, Phys. Status Solidi B 244, 100 (2007).

[13] V. Gadagkar, P.K. Maiti, Y. Lansac, A. Jagota, A.K. Sood, Phys. Rev. B 73, 085402 (2006).

[14] J. Arvanitidis, D. Christofilos, K. Papagelis, K.S. Andrikopoulos, T. Takenobu, Y. Iwasa, H. Kataura, S. Ves, G.A. Kourouklis, Phys. Rev. B 71, 125404 (2005).

[15] P. Puech, H. Hubel, D.J. Dunstan, R.R. Bacsa, C. Laurent, W.S. Bacsa, Phys. Rev. Lett. 93, 095506 (2004).

[16] J. Arvanitidis, D. Christofilos, Phys. Status Solidi B 244, 127 (2007).

[17] H. Kataura, Y. Maniwa, T. Kodama, K. Kikuchi, K. Hirahara, K. Suenaga, S. Iijima, S. Suzuki, Y. Achiba, W. Kratschmer, Synth. Met. 121, 1195 (2001).

[18] S. Bandow, M. Takizawa, K. Hirahara, M. Yudasaka, S. Iijima, Chem. Phys. Lett. 337, 48 (2001).

[19] D. Christofilos, J. Arvanitidis, J. Phys., Conf. Series 121, 162004 (2008).

[20] M.S. Dresselhaus, G. Dresselhaus, A. Jorio, A.G. Souza Filho, R. Saito, Carbon 40, 2043 (2002).

[21] A. Jorio, M.A. Pimenta, A.G. Souza Filho, R. Saito, G. Dresselhaus, M.S. Dresselhaus, New J. Phys. 5, 139 (2003).

[22] D.S. Bethune, G. Meijer, W.C. Tang, H.J. Rosen, W.G. Golden, H. Seki, C.A. Brown, M.S. de Vries, Chem. Phys. Lett. 179, 181 (1991).

[23] U.D. Venkateswaran, Phys. Status Solidi B 241, 3345 (2004).

[24] G. Raghuveer, M.Sc. Thesis, Lulea University, Sweden 2008.

[25] R. Pfeiffer, Ch. Kramberger, F. Simon, H. Kuzmany, V.N. Popov, H. Kataura, Eur. Phys. J. B 42, 345 (2004).

[26] R. Pfeiffer, H. Kuzmany, Ch. Kramberger, Ch. Schaman, T. Pichler, H. Kataura, Y. Achiba, J. Kurti, V. Zolyomi, Phys. Rev. Lett. 90, 225501 (2003). 